\title{
La Declaración de Cartagena y los Objetivos de Desarrollo Sostenible
}

\author{
The Cartagena Declaration and the Sustainable Development Goal \\ A Declaração de Cartagena e os Objetivos de Desenvolvimento Sustentável
}

\author{
Diana Cardenas ${ }^{1 *}$
}

Recibido para publicación: 1 de septiembre de 2019. Aceptado para publicación: 26 de octubre 2019. https://doi.org/10.35454/rncm.v2supl1.034

\begin{abstract}
Resumen
Los Objetivos de Desarrollo Sostenible (ODS) buscan terminar con todas las formas de hambre y desnutrición para 2030 y velar por el acceso de todas las personas, en especial los niños, a una alimentación suficiente y nutritiva durante todo el año. Sin embargo, las metas del ODS 2"Hambre Cero", y de los otros 16 ODS no incluyen la problemática de la desnutrición asociada a la enfermedad. La malnutrición (bajo peso, sobrepeso y obesidad, carencia de nutrientes) asociada a factores socio económicos (mala alimentación, hambre y pobreza) y la desnutrición asociada a la enfermedad tienen orígenes y mecanismos fisiopatológicos distintos; y por lo tanto, necesitan abordajes diferentes. La Declaración de Cartagena es un instrumento en que, por primera vez, el cuidado nutricional es elevado a categoría de derecho humano y puede ser considerado como una estrategia para dar visibilidad y llamar la atención de los formuladores de políticas públicas sobre la necesidad de avanzar en este campo. Al incluir este tipo de desnutrición dentro del abordaje global del problema de la malnutrición de las poblaciones estaríamos contribuyendo a lograr las metas de los ODS y en concreto al desarrollo sostenible de los países.
\end{abstract}

Palabras clave: objetivos de desarrollo sostenible, derechos humanos, malnutrición.

\section{Summary}

The Sustainable Development Goals (SDGs) seek to end all forms of hunger and malnutrition by 2030 and ensure the access of all people, especially children, to sufficient and nutritious food throughout the year. However, the goals of SDG 2 "Zero Hunger," and the other 16 SDGs do not include the problem of malnutrition associated with disease. Malnutrition (low weight, overweight and obesity, micronutrient deficiencies) associated with socioeconomic factors (poor diet, hunger and poverty) and malnutrition associated with disease have different pathophysiological origins and mechanisms and therefore need different approaches. The Cartagena Declaration is an instrument where nutritional care is defined for the first time as a human right and can be considered as a strategy to give visibility and draw the attention of public policy makers on the need to advance in this field. By including this type of malnutrition within the global approach to the problem of population malnutrition, we would be contributing to achieving the SDG targets and, in particular, to the sustainable development of countries.

Keyword: Sustainable Development Goals; Human Rights; Malnutrition.

\section{Resumo}

Os Objetivos de Desenvolvimento Sustentável (ODS) buscam acabar com todas as formas de fome e desnutrição até 2030 e garantir o acesso de todas as pessoas, especialmente crianças, a alimentos suficientes e nutritivos ao longo do ano. No entanto, os objetivos do ODS 2 "Fome Zero" e os outros 16 ODS não incluem o problema da desnutrição associado à doença. A desnutrição (baixo peso, sobrepeso e obesidade, carência de nutrientes) associada a fatores socioeconômicos (má alimentação, fome e pobreza) e a desnutrição associada à doença têm origens e mecanismos fisiopatológicos diferentes e, portanto, precisam de abordagens diferentes. A Declaração de Cartagena é um instrumento em que, pela primeira vez, o cuidado nutricional é elevado à categoria de direito humano e pode ser considerado uma estratégia para dar visibilidade e chamar a atenção dos formuladores de políticas públicas sobre a necessidade de avançar neste campo. Ao incluir esse tipo de desnutrição na abordagem global do problema da desnutrição populacional, estaríamos contribuindo para alcançar as metas dos ODS e, em particular, para o desenvolvimento sustentável dos países.

Palavras-chave: objetivos de desenvolvimento sustentável, direitos humanos, desnutrição.
Instituto de Investigación en Nutrición, Genética y Metabolismo, Facultad de Medicina, Universidad El Bosque, Bogotá, D.C., Colombia.
*Correspondencia: Diana Cardenas dianacardenasbraz@gmail.com 


\section{INTRODUCCIÓN}

Según las Naciones Unidas, el desarrollo sostenible se define como el "desarrollo capaz de satisfacer las necesidades del presente sin comprometer la capacidad de las futuras generaciones para satisfacer sus propias necesidades. El desarrollo sostenible exige esfuerzos concertados para construir un futuro inclusivo, sostenible y resiliente para las personas y el planeta." ${ }^{(1)}$. Tiene en cuenta tres elementos básicos: el crecimiento económico, la inclusión social y la protección del medio ambiente. Estos elementos están interrelacionados y son esenciales para lograr el bienestar de las personas y las sociedades ${ }^{(1)}$. El hambre extrema y la malnutrición siguen siendo un enorme obstáculo para el desarrollo sostenible. El hambre y la malnutrición hacen que las personas sean menos productivas y más propensas a sufrir enfermedades, por lo que no suelen ser capaces de aumentar sus ingresos y mejorar sus medios de vida.

Con el objetivo de orientar los esfuerzos de los países para lograr un desarrollo sostenible, en septiembre de 2015, más de 150 jefes de Estado y de Gobierno se reunieron en la histórica Cumbre del Desarrollo Sostenible en la que aprobaron la Agenda 2030 de Naciones Unidas. Esta Agenda contiene 17 objetivos y las 169 metas de aplicación universal que, desde el 1 de enero de 2016, rigen los esfuerzos de los países para lograr un mundo sostenible ${ }^{(1)}$. Los Objetivos de Desarrollo Sostenible (ODS) reemplazan a los Objetivos de Desarrollo del Milenio (ODM), y buscan lograr aquellas metas que no fueron conseguidas. Lo innovador de los ODS es que insta a todos los países, sin distinción alguna, a adoptar una serie de medidas para promover el desarrollo al tiempo que protegen el planeta. Reconocen que las iniciativas para acabar con temas como el hambre deben ir de la mano de estrategias que favorezcan el crecimiento económico y aborden una serie de necesidades sociales, entre las que cabe señalar la educación, la salud, la protección social y las oportunidades de empleo, a la vez que luchan contra el cambio climático y promueven la protección del medio ambiente. Los ODS no son jurídicamente obligatorios, sin embargo, los países se fijan unas metas que deben logra a través de acciones y políticas nacionales.

\section{OBJETIVO 2: HAMBRE CERO}

Poner fin al hambre, lograr la seguridad alimentaria y la mejora de la nutrición y promover la agricultura soste- nible son las metas del objetivo 2 "Hambre cero". Un mundo sin hambre y en buen estado nutricional puede tener un impacto positivo en la economía, así como en la salud, la educación, la igualdad y el desarrollo social generales. Este objetivo de Hambre Cero es un punto central en la construcción de un futuro mejor para todos los países. El hambre y la desnutrición al frenar el desarrollo humano, impediría que se logren los otros Objetivos de Desarrollo Sostenible, como la educación, la salud y la igualdad de género ${ }^{(2)}$ (Tabla 1, Figura 1).

\section{LA DESNUTRICIÓN ASOCIADA A LA ENFERMEDAD Y LOS ODS}

La desnutrición asociada a la enfermedad, un tipo específico de desnutrición causada por una enfermedad concomitante, es altamente prevalente en todos los países del mundo ${ }^{(3,4)}$. En Latinoamérica la prevalencia de desnutrición al ingreso hospitalario es mayor que en otras regiones del mundo, según una revisión de la literatura es de $40 \%$ a $60 \%(5)$, y aumenta con la duración de la estancia hospitalaria. La desnutrición relacionada con la enfermedad se asocia a una calidad de vida reducida de los pacientes y un aumento de las comorbilidades infecciosas y no infecciosas, la duración de la estadía y los costos que imponen una considerable carga económica y de salud a esos países ${ }^{(6,7)}$.

El riesgo de desnutrición en el momento de la admisión se ha correlacionado con varios factores entre ellos las alteraciones metabólicas, el impacto de la enfermedad en los requerimientos nutricionales, la disminución de la ingesta de alimentos, los problemas de organización y la falta de sensibilización y educación médica. Consideramos que cualquiera sea la razón, la mayor prevalencia de desnutrición relacionada con la enfermedad en Latinoamérica podría verse influenciada por la mayor prevalencia de hambre y desnutrición de la población en general. Esto significa que las condiciones socioeconómicas y las condiciones de salud pública (acceso y cobertura en salud) pueden influir en la mayor prevalencia de riesgo nutricional hospitalario en la región.

La carga de la desnutrición se investigó en Colombia en pacientes hospitalizados con enfermedad cardiovascular o pulmonar en un estudio multicéntricos realizado por Ruiz et al. ${ }^{(8)}$. Este estudio mostró que el riesgo de desnutrición detectado con la herramienta Malnutrition Screening Tool (MST) se asoció a un aumento de 1,6 días en el promedio de la estancia hospitalaria, con un incremento relativo de $30,13 \%$ en el 
Tabla 1. Metas del Objetivo 2: Hambre Cero

2.1 Para 2030, poner fin al hambre y asegurar el acceso de todas las personas, en particular los pobres y las personas en situaciones vulnerables, incluidos los lactantes, a una alimentación sana, nutritiva y suficiente durante todo el año

2.2 Para 2030, poner fin a todas las formas de malnutrición, incluso logrando, a más tardar en 2025, las metas convenidas internacionalmente sobre el retraso del crecimiento y la emaciación de los niños menores de 5 años, y abordar las necesidades de nutrición de las adolescentes, las mujeres embarazadas y lactantes y las personas de edad

2.3 Para 2030, duplicar la productividad agrícola y los ingresos de los productores de alimentos en pequeña escala, en particular las mujeres, los pueblos indígenas, los agricultores familiares, los pastores y los pescadores, entre otras cosas mediante un acceso seguro y equitativo a las tierras, a otros recursos de producción e insumos, conocimientos, servicios financieros, mercados y oportunidades para la generación de valor añadido y empleos no agrícolas

2.4 Para 2030, asegurar la sostenibilidad de los sistemas de producción de alimentos y aplicar prácticas agrícolas resilientes que aumenten la productividad y la producción, contribuyan al mantenimiento de los ecosistemas, fortalezcan la capacidad de adaptación al cambio climático, los fenómenos meteorológicos extremos, las sequías, las inundaciones y otros desastres, y mejoren progresivamente la calidad del suelo y la tierra.

2.5 Para 2020, mantener la diversidad genética de las semillas, las plantas cultivadas y los animales de granja y domesticados y sus especies silvestres conexas, entre otras cosas mediante una buena gestión y diversificación de los bancos de semillas y plantas a nivel nacional, regional e internacional, y promover el acceso a los beneficios que se deriven de la utilización de los recursos genéticos y los conocimientos tradicionales y su distribución justa y equitativa, como se ha convenido internacionalmente.

Para ello se deberá:

2.a Aumentar las inversiones, incluso mediante una mayor cooperación internacional, en la infraestructura rural, la investigación agrícola y los servicios de extensión, el desarrollo tecnológico y los bancos de genes de plantas y ganado a fin de mejorar la capacidad de producción agrícola en los países en desarrollo, en particular en los países menos adelantados.

2.b Corregir y prevenir las restricciones y distorsiones comerciales en los mercados agropecuarios mundiales, entre otras cosas, mediante la eliminación paralela de todas las formas de subvenciones a las exportaciones agrícolas y todas las medidas de exportación con efectos equivalentes, de conformidad con el mandato de la Ronda de Doha para el Desarrollo.

2.c Adoptar medidas para asegurar el buen funcionamiento de los mercados de productos básicos alimentarios y sus derivados y facilitar el acceso oportuno a información sobre los mercados, en particular sobre las reservas de alimentos, a fin de ayudar a limitar la extrema volatilidad de los precios de los alimentos.

Fuente: https://www.un.org/sustainabledevelopment/es/.

costo promedio asociado a la hospitalización. También se asoció a un aumento en el riesgo de mortalidad hasta 30 días después del alta hospitalaria.

Numerosos estudios han demostrado que el cuidado nutricional puede mejorar los resultados clínicos y reducir los costos de atención médica en diferentes áreas de la enfermedad, como en los pacientes en estado crítico $^{(9,10)}$, pancreatitis ${ }^{(11)}$, adultos mayores ${ }^{(12)}$, pacientes con disfagia ${ }^{(13)}$ y pacientes con enfermedad obstructiva crónica $^{(14)}$. A pesar de esta evidencia, la desnutrición relacionada con la enfermedad no se detecta a menudo y, por lo tanto, no se trata en los hospitales, no se generan alertas ni produce inquietud en los responsables de formular las políticas. Son escasos los países que tienen legislaciones y políticas públicas en este tema. A esto se suma que ninguna de las metas del ODS 2 , ni de cualquiera de los otros 16 ODS, menciona la desnutrición asociada a la enfermedad (Tabla 1). Esto quiere decir que los gobiernos se centrarán en abordar la desnutrición y la seguridad alimentaria sin tener en cuenta este tipo específico de desnutrición asociada a la enfermedad.

\section{EL ODS 2 Y LA DECLARACIÓN DE CARTAGENA}

Para incluir la problemática de la desnutrición asociada a la enfermedad en la agenda política nacional e internacional y aumentar la probabilidad de que se formulen políticas para combatir este problema, en particular en al ámbito hospitalario, se necesita como primera medida dar visibilidad al problema y generar conciencia sobre su importancia. La Declaración de Cartagena es un instrumento donde, por primera vez, el cuidado nutricional se eleva a categoría de derecho humano. Aunque esta Declaración compromete únicamente a las sociedades a trabajar en su defensa, es un primer paso para dar visibilidad y llamar la atención de los formuladores de políticas 


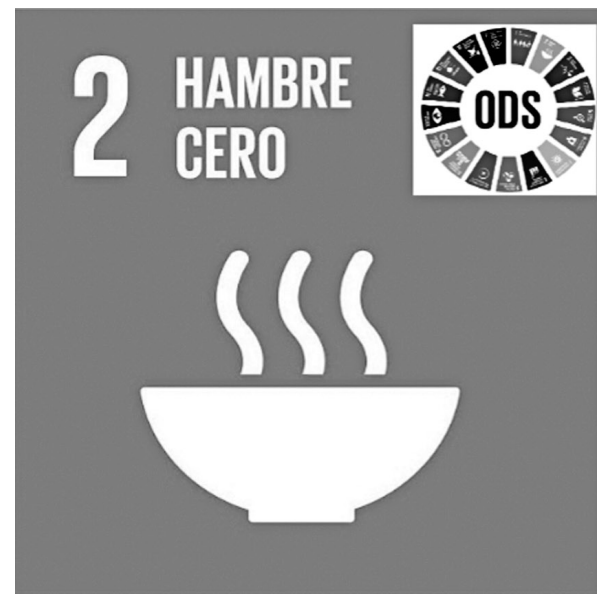

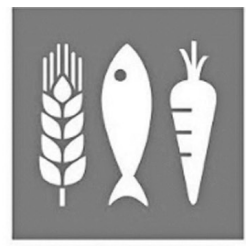

2.1. Hambre cero y asegurar alimentación $3 S$

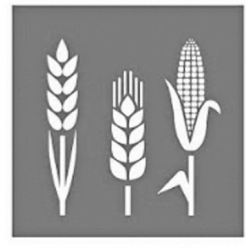

2.5. Mantenimiento de la diversidad genética

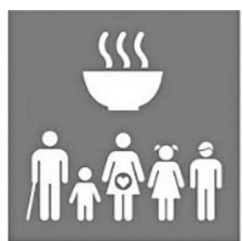
2.2. Poner fin a todas las formas
de malnutrición

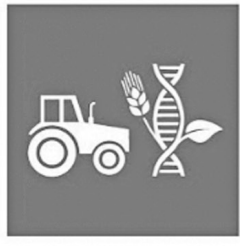

2.A. Inversión en infraestructuras, investigación y desarrollo agrario

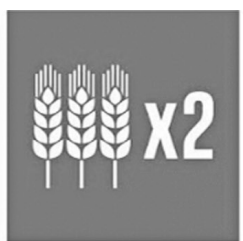

2.3. Duplicar productividad agricola a pequeña escala

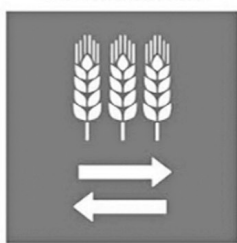

2.8. Estabilidad mercados agropecuarios mundiales

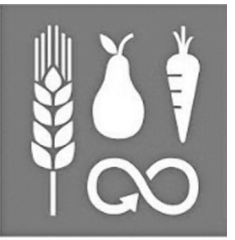

2.4. Prácticas agricolas sostenibles y resilentes

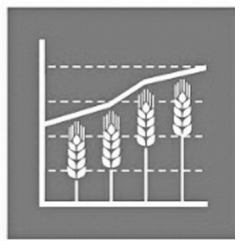

2.c. Control volatilidad de

\section{NUTRITION AND THE SDGs}

CENTRAL TO THE 2030 AGENDA

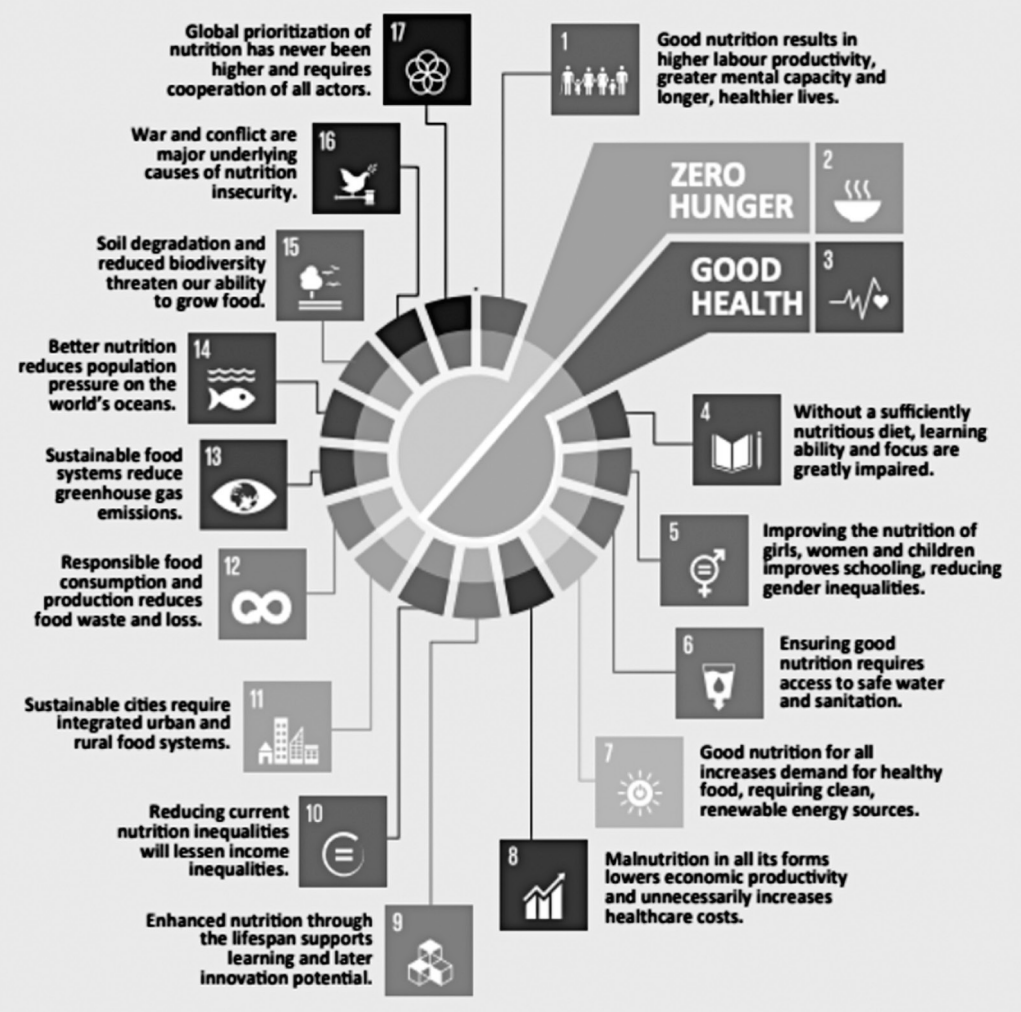

UNITED NATIONS DECADE OF

ACTION ON NUTRITION

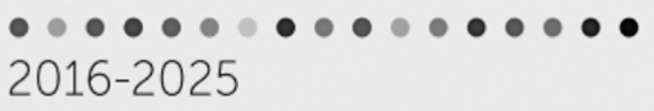

Figura 1. Incluir la desnutrición asociada a la enfermedad y promover el cuidado nutricional en el ámbito clínico contribuye al desarrollo sostenible de los países. Adaptado de: https://www.un.org/sustainabledevelopment/es/. 
A

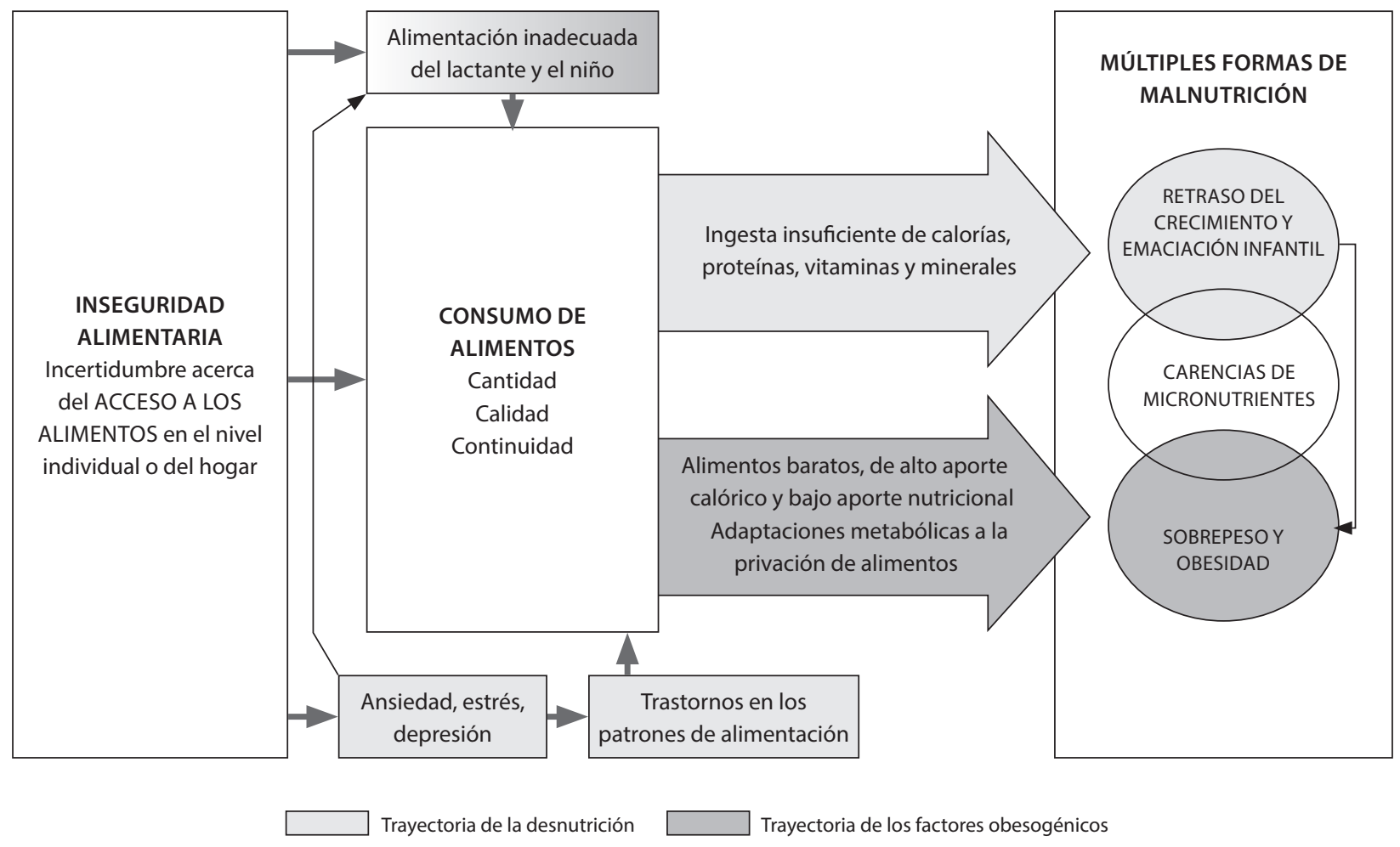

B

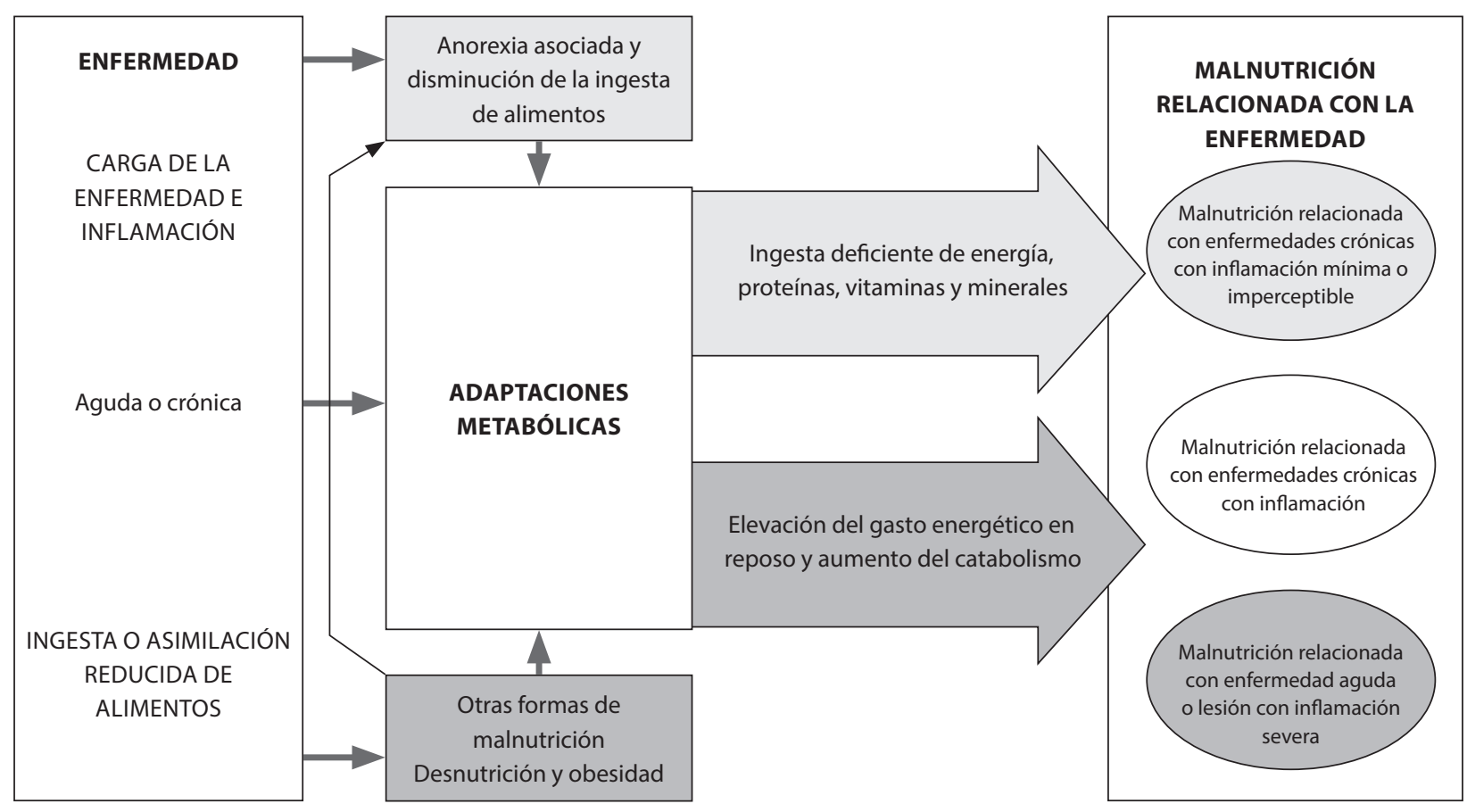

Figura 2. A. Trayectoria del acceso inadecuado a los alimentos a las múltiples formas de malnutrición, según la FAO, 2018(15). B. Trayectoria de la enfermedad a las diversas formas de desnutrición. Figura basada en la definición y clasificación de la desnutrición ${ }^{(4)}$. 
públicas. Elevar el cuidado nutricional al rango de derecho humano servirá como una estrategia para que al evaluar y buscar soluciones al problema de desnutrición de la población también se tenga en cuenta la desnutrición asociada a la enfermedad. Esto es esencial si tenemos en cuenta que la malnutrición (bajo peso, sobrepeso y obesidad) asociada a factores socio económicos (mala alimentación, hambre y pobreza) y la desnutrición asociada a la enfermedad tienen orígenes y mecanismos fisiopatológicos distintos; y por lo tanto, necesitan un abordaje diferente (Figura 2).

De esta forma, al hacer visible el problema y luchar por un cuidado nutricional para todos los pacientes, estaríamos contribuyendo al desarrollo sostenible de los países.

\section{CONCLUSIÓN}

Cuando las sociedades científicas y los profesionales de la salud asumen la defensa del derecho al cuidado nutricional promueven la visibilidad de esta problemática. El objetivo es lograr que la desnutrición asociada a la enfermedad se incluya dentro del abordaje global del problema de la malnutrición de las poblaciones. De esta forma, desde la ciencia de la nutrición clínica, se está contribuyendo a lograr las metas de los ODS y en concreto al desarrollo sostenible de los países.

\section{Financiación}

El presente artículo no tuvo financiación.

\section{Conflicto de intereses}

El autor declara no tener conflicto de intereses.

\section{Referencias bibliográficas}

1. Naciones Unidas. Objetivos de Desarrollo Sostenible. [Internet]. [consultado el 29 ocubre 2015]. Disponible en: https://www.un.org/sustainabledevelopment/es/.

2. Naciones Unidas. Objetivos de Desarrollo Sostenible. ODS 2: Hambre Cero. [Internet]. [consultado el 29 ocubre 2015]. Disponible en https://www.un.org/sustainabledevelopment/ es/wpcontent/uploads/sites/3/2016/10/2_Spanish_Why_ it_Matters.pdf.
3. Sobotka L. Editor. Basics in clinical nutrition. 4th ed. Galen , Prague, 2012.

4. Cederholm CT, Barazzoni R, Austin P, Ballmer P, Biolo G, Bischoff SC, et al. ESPEN guidelines on definitions and terminology of clinical nutrition. Clin Nutr. 2017;36(1):49-64. doi: 10.1016/j.clnu.2016.09.004.

5. Correia MITD, Perman MI, Waitzberg DL. Hospital malnutrition in Latin America: A systematic review. Clin Nutr. 2017;36:958-67.

6. Norman K, Pichard C, Lochs H, Pirlich M. Prognostic impact of disease-related malnutrition. Clin Nutr. 2008;27:5-15.

7. Rodriguez-Manas, Abizanda P, Barcons N, Lizán L. Malnutrition in Institutionalized and Community-Dwelling Older Adults in Spain: Estimates of Its Costs To the National Health System. Value Health. 2014;17(7):A507.

8. Ruiz AJ, Buitrago G, Rodríguez N, Gómez G, Sulo S, Gómez C, Partridge J, Misas J, Dennis R, Alba MJ, Chaves-Santiago $\mathrm{W}$, Araque C. Clinical and economic outcomes associated with malnutrition in hospitalized patients. Clin Nutr. 2019;38(3):1310-6 doi: 10.1016/j.clnu.2018.05.016

9. Doig GS, Heighes PT, Simpson F, Sweetman EA, Davies AR. Early enteral nutrition, provided within $24 \mathrm{~h}$ of injury or intensive care unit admission, significantly reduces mortality in critically ill patients: a meta-analysis of randomised controlled trials. Intensive Care Med. 2009;35(12):2018-27.

10. Visser J, Labadarios D, Blaauw R. Micronutrient supplementation for critically ill adults: a systematic review and metaanalysis. Nutrition. 2011;27(7-8):745-58.

11. McClave SA, Chang WK, Dhaliwal R, Heyland DK. Nutrition support in acute pancreatitis: a systematic review of the literature. JPEN J Parenter Enteral Nutr. 2006;30(2):143-56.

12. Deutz NE, Matheson EM, Matarese LE, Luo M, Baggs GE, Nelson JL, et al. Readmission and mortality in malnourished, older, hospitalized adults treated with a specialized oral nutritional supplement: a randomized clinical trial. Clin Nutr. 2016;35(1):18-26.

13. Cook IJ. Treatment of oropharyngeal dysphagia. Curr Treat Options Gastroenterol. 2003;6(4):273-81.

14. Snider JT, Jena AB, Linthicum MT, Hegazi RA, Partridge JS, LaVallee C, et al. Effect of hospital use of oral nutritional supplementation on length of stay, hospital cost, and 30-day readmissions among medicare patients with COPD. Chest. 2015;147(6):1477-84.

15. Food and Agriculture Organization of the United Nations. The state of food security and nutrition in the world. [Internet]. Roma 2018. (Consultado el :5 september 2019). Disponible en: http://www.fao.org/3/I9553ES/i9553es.pdf. 